Esta revista forma parte del acervo de la Biblioteca Jurídica Virtual del Instituto de Investigaciones Jurídicas de la UNAM

\title{
Conexión entre medios de información y preferencias electorales
}

\author{
Jesús Cantú* \\ Everardo Díaz** \\ Rocío Galarza*** \\ Alma Rosa Suárez****
}

\section{Sumario:}

I. Introducción

II. Medios y equidad en las contiendas democráticas

III. ¿Influyen los medios en el voto?

IV. Niveles de información y decisión política

V. Cobertura mediática

VI. Medios de información y preferencias electorales

VII. Preferencia electoral y medios de información

VIII. Grupos de enfoque

IX. Conclusiones

X. Fuentes consultadas

* Director de la Maestría en Análisis Político y Medios de Información de EGAP Gobierno y Política Pública; coordinador del Proyecto Observatorio de Medios y Opinión Pública EGAP.

** Candidato a doctor en Ciencia Política; investigador del Proyecto Observatorio de Medios y Opinión Pública EGAP.

*** Maestra en Análisis Político y Medios de Información; investigadora del Proyecto Observatorio de Medios y Opinión Pública EGAP.

**** Maestra en Análisis Político y Medios de Información: investigadora del Proyecto Observatorio de Medios y Opinión Pública EGAP.

D. R. () 2013. PNUD-UNAM, Instituto de Investigaciones Jurídicas. 
Esta revista forma parte del acervo de la Biblioteca Jurídica Virtual del Instituto de Investigaciones Jurídicas de la UNAM www.juridicas.unam.mx

\section{Resumen:}

El presente artículo tiene como propósito mostrar los resultados de la investigación realizada en torno a aspectos cuantitativos y cualitativos del desempeño de los medios de comunicación masiva en México durante la campaña electoral de 2012 y el impacto de los mismos en la opinión pública. En primer lugar, se presenta la evidencia por el lado de la oferta informativa, a partir del análisis de contenido de medios que se hizo en el Observatorio de Medios EGAP, en el mismo se encuentran importantes diferencias cuantitativas y cualitativas en términos de tono, volumen de notas, y estilos periodísticos, entre los medios impresos y las televisoras. Por el lado del público elector, se utiliza la evidencia arrojada por las encuestas nacionales levantadas por el Tecnológico de Monterrey, respecto a preferencia electoral y preferencia de usos de medios. Finalmente, se presentan algunos resultados relevantes de los grupos de enfoque realizados respecto a la percepción pública de la cobertura de medios sobre la campaña.

Palabras clave: elecciones en México 2012, Observatorio de Medios, preferencias electorales, cobertura mediática, opinión pública. 


\section{Introducción}

Las democracias electorales modernas, para poder ser consideradas tales, requieren garantizar la igualdad formal en el acceso a los recursos políticos. El acceso a los medios de comunicación por parte de los partidos políticos se ha convertido en un requisito, y el caso mexicano se ha considerado un éxito, al menos en lo que se refiere al acceso a los espacios publicitarios. Sin embargo, la igualdad política también requiere que las fuentes de información, en su conjunto, presenten puntos de vista diversos y representativos de las fuerzas políticas. Es este aspecto de posibles sesgos por parte de los medios de comunicación, y su impacto en las contiendas electorales, el objeto central de este trabajo.

La intención de este artículo es mostrar los resultados de la investigación realizada en torno a aspectos cuantitativos y cualitativos del desempeño de los medios de comunicación masiva en México durante la campaña electoral de 2012 y el impacto del mismo en la opinión pública. Lo anterior se efectuó con base en el análisis de contenido de medios que hizo el Observatorio de Medios de la Escuela de Graduados en Administración Pública (EGAP) del Instituto Tecnológico de Estudios Superiores de Monterrey; a partir de las encuestas nacionales levantadas, con motivo de la elección presidencial, en relación con preferencia electoral y preferencia de usos de medios; así como tomando en cuenta los resultados relevantes de los grupos de enfoque desarrollados respecto a la percepción pública de la cobertura de medios sobre la campaña.

\section{Medios y equidad en las contiendas democráticas}

Desde una perspectiva formal y jurídica, Dahl $^{1}$ considera a la igualdad política con un objetivo inseparable del ideal democrático. Las democracias electorales modernas, para poder ser tales, deben distribuir de

1 Dahl, Robert A., La igualdad política, Buenos Aires, Fondo de Cultura Económica de Argentina, 2008. 
Esta revista forma parte del acervo de la Biblioteca Jurídica Virtual del Instituto de Investigaciones Jurídicas de la UNAM

forma justa el acceso a los recursos políticos. ${ }^{2}$ Entre estos recursos, el acceso a medios de comunicación es quizá uno de los más decisivos en esta era de comunicación masiva. Para poder aspirar a una democracia, es necesario asegurarse que la diversidad de opiniones y perspectivas políticas reciban espacios más o menos comparables y equilibrados en los medios masivos de comunicación. Este ideal de pluralismo en las perspectivas políticas puede lograrse de una forma externa, a través de la existencia de una pluralidad de medios u organizaciones que reflejan los puntos de vista de diferentes grupos o tendencias en la sociedad. ${ }^{3}$ También puede lograrse a través de un pluralismo interno, que se alcanza "en cada medio u organización en lo individual", ${ }^{4}$ a través de un contenido balanceado. Como se mostrará en este estudio, para el caso mexicano el pluralismo interno en los medios de comunicación masivos es casi inexistente, y el pluralismo externo se ve limitado a la prensa escrita. Esto plantea cuestionamientos importantes acerca de la calidad de nuestra democracia electoral, y constituyen un tema necesario de análisis y reflexión.

\section{III. ¿Influyen los medios en el voto?}

Ahora bien, la equidad en el acceso a los recursos de comunicación política no es deseable sólo por razones formales o morales. Existe un amplio debate en la literatura acerca del grado y alcance de la influencia que tienen los medios de comunicación en la formación de opiniones políticas, que llevan a la decisión de voto. La mayor parte de los estudios coinciden en que los medios de comunicación tienen algún grado de influencia en la formación de opiniones y percepciones de alcance político. Sin embargo, existen profundos desacuerdos respecto al peso que tienen los medios en la consideración final de voto. Para propósito de este trabajo, el marco conceptual que se emplea se debe sobre todo a dos grupos teóricos claramente diferenciados.

2 Diamond, Larry y Morlino, Leonardo, "An Overview”, The Quality of Democracy. Journal of Democracy, 2004.

3 Hallin, Daniel C. y Mancini, Paolo, Comparing Media Systems. Three Models of Media and Politics, Cambridge, USA, Cambridge University Press, 2004.

4 Ibidem, p. 29. 
Esta revista forma parte del acervo de la Biblioteca Jurídica Virtual del Instituto de Investigaciones Jurídicas de la UNAM

En primer lugar, se tienen las teorías de establecimiento de agenda (agenda setting) que constituyen una corriente muy influyente dentro de los estudios de comunicación política. A partir de la labor pionera de McCombs y Shaw, ${ }^{5}$ quienes en su estudio presentaron algunas de las ideas centrales sobre las campañas presidenciales estadounidenses de 1968 y 1972, se ha creado una escuela que se enfoca en el manejo mediático de los contenidos. En las teorías de agenda setting, los medios de comunicación determinan los temas considerados centrales o importantes, concentrando la atención del público, en un proceso que es necesariamente intencional. Es decir, los medios no reflejan la realidad, sino que escogen y filtran la información que llega a las audiencias, a través de reglas de decisiones imperfectas y no siempre claras. De acuerdo con esta teoría, los medios de comunicación no son muy efectivos en imponer opiniones, pero sí son muy influyentes en determinar acerca de qué piensa el público. ${ }^{6}$

La evolución natural de las teorías de agenda setting permitió agregar y enfatizar los aspectos de comunicación que se refieren a la contextualización y al marco interpretativo de decodificación del mensaje. En esta perspectiva, los medios no sólo atribuyen importancia o jerarquía a las notas, sino que también nos proporcionan el marco conceptual para el entendimiento y decodificación del mensaje. Esto es, el framing y el priming ${ }^{8}$ se refieren al cómo se presenta información (bajo qué marco conceptual, valorativo o ideológico), una vez que se ha seleccionado la información que se presenta o resalta (el qué, objeto de estudio de la agenda setting). La teoría evolucionada de agenda setting, con las aportaciones sobre los efectos de framing y priming, es el principal soporte de los estudios empíricos que se presentan en este trabajo.

De forma complementaria, se toman también elementos de las llamadas "teorías de cultivación", 9 en las cuales los efectos de los medios de comunicación en la audiencia son de largo plazo: construyen lenta-

\footnotetext{
5 McCombs, M. E. y Shaw, D., “The Agenda-Setting Function of Mass Media”, POQ, 36, 1972, pp. 176-187.

6 Cohen, B., The Press and Foreign Policy, Princeton, NJ, Princeton University Press, 1963.

7 Véase Fairhurst, G. y Star, R., The art of Framing, San Francisco, Jossey-Bass, 1996.

8 Véase Domke, D. et al., "Media Priming Effects: Accessibility, Association, and Activation", Communications Abstracts, 21( 6), 1998.

9 Gerbner, G., y Gross, L., “The Scary World of TV's Heavy Viewer”, Psychology Today, 10(4), 1976, pp. 41-89.
} 
Esta revista forma parte del acervo de la Biblioteca Jurídica Virtual del Instituto de Investigaciones Jurídicas de la UNAM

mente una "imagen", un conjunto conceptual y de valores alrededor de un tema o una persona. Esto no ocurre a partir de un evento aislado; más bien, es un fenómeno de largo plazo y acumulativo. Este marco conceptual es especialmente útil para los fines de televisión, medio para el que se presupone que el nivel de exposición está relacionado directamente con la magnitud del efecto en las preferencias.

\section{Niveles de información y decisión política}

La segunda línea teórica de gran importancia para este estudio es el impacto que tienen los distintos niveles de información del público en la toma de decisiones formales colectivas. La influencia de los medios en el público está mediada por una multitud de factores, entre los que destacan el nivel educativo, los hábitos de uso de nuevas tecnologías (fuertemente ligados a la edad y al nivel de ingreso), y posiblemente la influencia del sistema electoral en los estilos de campaña electoral. Este corpus teórico, que se puede traducir como la "brecha informativa" ("knowledge gap"), ${ }^{10}$ conceptualiza al individuo como decisor racional, cuya selección de voto está determinada primordialmente por la información disponible. Los individuos obtienen información de una multitud de fuentes; sin embargo, no todos tienen el tiempo ni los recursos para poder dar seguimiento detallado a todos los eventos, en suma, no todos tienen los mismos niveles de información. Por lo tanto, en lugar de investigar exhaustivamente toda la información política disponible, se utilizan algunos elementos o "pistas" generales del debate para informarse, y las complementan con creencias previas u opiniones de amigos o familiares. En este modelo, los medios pueden influir en los individuos con menores niveles de información, ya que ellos cuentan con menos herramientas para poder discernir y evaluar la información que reciben y contrastarla con otras fuentes o con información "de calidad". De hecho, en las democracias modernas es perfectamente plausible el que la propaganda informativa se concentre en un segmento relativamente reducido del electoral, y que aún así determine el resultado de una contienda electoral.

10 Tichenor, P. J. et al., "Mass Media Flow and Differential Growth in Knowledge", Public Opinion Quarterly, 34, Colombia University Press, 1970. 


\section{Cobertura mediática}

En la sección de la investigación referente a la cobertura mediática, lo que se realizó fue un análisis de la información que medios impresos y de televisión en México presentaron sobre las elecciones presidenciales de 2012. Esto se hizo a través de técnicas de análisis de contenido que combinaron aspectos cuantitativos y cualitativos, con ello se pretendió detectar las tendencias, sesgos y preferencias expresadas en los principales medios de comunicación del país.

Los medios estudiados fueron los dos principales noticieros de televisión abierta en México, "El Noticiero" con Joaquín López Dóriga/Televisa, y "Hechos", con Javier Alatorre/TV Azteca, así como tres diarios de circulación nacional: El Universal, La Jornada, y Reforma. Todas las notas aparecidas en los noticieros, así como en la portada y en las secciones relevantes en el caso de los periódicos, se sujetaron a un primer proceso de clasificación estrictamente descriptivo (fecha, medio, tema, formato, duración/extensión, localización dentro del orden de la noticia, uso de recursos, descripción general de contenido).

A continuación, las notas pasaron a una segunda etapa de análisis y evaluación, en donde se establecieron y definieron: $a$ ) actores; b) tratamiento de actores; $c$ ) tono; $d$ ) aspectos de framing, tales como posicionamiento del medio; uso de apelaciones emocionales, morales o personales; identificación de conflicto; tratamiento de causas y consecuencias; atribución de responsabilidades, etcétera. Esto último con base en trabajos previos hechos por Semetko y Valkunberg ${ }^{11}$ y Esser y D’Angelo. ${ }^{12}$

La temporalidad de las notas de contenido político electoral que se estudiaron abarcó un periodo de poco más de tres meses, a partir del 31 de marzo de 2012, y extendiéndose hasta el 2 de julio del mismo año. Asimismo, se utilizó información general —sin análisis de framing - de las notas sobre los candidatos (sólo las de portada para el

11 Véase Semetko y Valkunberg Framing, "European Politics: a Content Analysis of Press and Television News", Journal of Communication, 50 (2), 2006.

12 Véase Esser y D’Angelo, "Framing the Press and Publicity Process in U.S., British and German General Election Campaings: a Comparative Study of Metacoverage", The Harvard International Journal of Press/Politics 11(44), 2006. 
Esta revista forma parte del acervo de la Biblioteca Jurídica Virtual del Instituto de Investigaciones Jurídicas de la UNAM

caso de los periódicos) aparecidas desde el 1 de abril de 2011, esto es, un año antes del inicio formal de la campaña presidencial.

Para los periódicos se procesaron 5564 notas electorales aparecidas en primera plana o en la sección de política/nacional respectivas, una media de casi 80 notas por día de cobertura analizada. En lo que respecta a la televisión, se procesaron 722 segmentos cuyo tema central era la campaña. Como resultado de la investigación se encontró que hubo diferencias marcadas entre la cobertura de la campaña electoral que hicieron medios impresos y medios electrónicos.

Se establecieron tres categorías para determinar la naturaleza del mensaje que presentaba cada medio. ${ }^{13}$ Para medios impresos y electrónicos se encontró una tendencia general al presentar notas que fueron denominadas de "conducto", por su carácter descriptivo, en el que el medio actuó meramente como transmisor de información. En su mayoría, los medios reprodujeron lo que decían o hacían los contendientes, partidos políticos u órganos electorales, dando particular énfasis a los eventos de campaña orquestados por cada candidato. Sin embargo, esto tuvo predominio proporcionalmente en la televisión, como se aprecia en la tabla 1.

En cambio, los periódicos sí tuvieron un mayor porcentaje de notas llamadas de "estrategia", en las que el medio buscaba intervenir como un actor más del escenario político, presentando información que se perfilaba a favor o en contra de uno u otro candidato. En los noticieros también se identificaron notas con esta intención, pero en menor medida. Mientras que en periódicos la mayoría de las notas editoriales sí fueron consideradas como estratégicas, en la televisión los editoriales se dedicaban más a la burla de los acontecimientos políticos que a intentar posicionarse respecto a un candidato $\mathrm{u}$ otro.

${ }_{13}$ Con base en las categorías desarrolladas por Esser y D’Angelo para estudiar la metacobertura en la cobertura de las elecciones en tres países: Estados Unidos, Gran Bretaña y Alemania. 
Esta revista forma parte del acervo de la Biblioteca Jurídica Virtual del Instituto de Investigaciones Jurídicas de la UNAM

Tabla 1. Meta cobertura en noticieros

\begin{tabular}{|l|c|c|c|}
\hline & Conducto & Estrategia & Accountability \\
\hline Alatorre & 264 & 38 & 0 \\
\hline López Dóriga & 377 & 43 & 0 \\
\hline
\end{tabular}

Tabla 2. Meta cobertura en periódicos

\begin{tabular}{|l|c|c|c|}
\hline & Conducto & Estrategia & Accountability \\
\hline El Universal & 1140 & 513 & 9 \\
\hline La Jornada & 1258 & 413 & 10 \\
\hline Reforma & 1659 & 533 & 29 \\
\hline
\end{tabular}

Como se observa en las tablas anteriores, se encontraron pocas notas de investigación a profundidad surgidas de los propios medios sobre cuestiones relativas a principios democráticos, tales como transparencia, rendición de cuentas, equidad, entre otros, las cuales eran clasificadas como de accountability. De hecho, en la televisión no se detectó una sola nota con estas características, en estos términos, y considerando también esfuerzos de los medios impresos por dar espacio a la difusión de las propuestas de los candidatos sobre temas específicos, puede decirse que la cobertura de periódicos fue de mejor calidad que la de los noticieros. Esto se estima así porque se considera que la labor de los medios de comunicación durante la campaña debe fomentar la discusión sobre las plataformas y políticas públicas de los candidatos, así como escudriñar sus antecedentes y habilidad para llevar a cabo sus propuestas.

Un tema que desde antes de que arrancara la campaña se anticipaba como relevante y que resultó importante en el desarrollo de la misma fue el volumen de cobertura. En coincidencia con lo que reportó el monitoreo del Instituto Federal Electoral - elaborado por la UNAM - en términos de cantidad de notas, se encontró que durante la campaña los noticieros mantuvieron un relativo balance en la presencia de los candidatos en su espacio. En lo que respecta a la prensa escrita, Enrique Peña Nieto recibió más atención que sus contrincantes, pero con un tono predominantemente negativo. 
Esta revista forma parte del acervo de la Biblioteca Jurídica Virtual del Instituto de Investigaciones Jurídicas de la UNAM

Tabla 3. Número de notas por candidato, todos los periódicos

\begin{tabular}{|l|c|c|c|c|}
\hline & Abril & Mayo & Junio & Julio \\
\hline JVM & 33 & 641 & 586 & 22 \\
\hline AMLO & 26 & 576 & 864 & 32 \\
\hline EPN & 32 & 760 & 1017 & 37 \\
\hline Quadri & 20 & 186 & 212 & 8 \\
\hline
\end{tabular}

Tabla 4. Número de notas por candidato, ambos noticieros

\begin{tabular}{|l|c|c|c|c|}
\hline & Abril & Mayo & Junio & Julio \\
\hline JVM & 29 & 79 & 72 & 6 \\
\hline AMLO & 26 & 77 & 100 & 8 \\
\hline EPN & 32 & 85 & 100 & 14 \\
\hline Quadri & 22 & 57 & 58 & 4 \\
\hline
\end{tabular}

No obstante, resulta muy importante enfatizar lo que sucedió previo al proceso electoral, periodo en el que el candidato del PRI, en su calidad de gobernador del Estado de México, tuvo fuerte presencia en ambos noticieros. Aunque las notas con un estilo promocional sobre administraciones estatales no fueron exclusivas de Peña Nieto, sí fue el personaje que predominó en ese estilo de cobertura, la cual resultó desproporcionada para cualquier mandatario estatal, incluyendo al jefe de gobierno del Distrito Federal, Marcelo Ebrard, y comparable hasta con la presencia en televisión del presidente de la República, Felipe Calderón. Esta tendencia no se detectó en medios impresos.

Otro aspecto que se debe mencionar es el tono con el que se manejó la cobertura sobre los diferentes candidatos. En televisión, ésta fue en su mayoría de tono neutro (oscilando entre el 69 y el $88 \%$ de las notas según el noticiero y el candidato del que se trataba) y en menor medida negativo hacia los candidatos, siendo raras las notas que presentaban un tono favorable (entre 0 y $5 \%$ según el caso). Los periódicos, por su parte, usaron un tono más valorativo en su cobertura a los candidatos presidenciales, predominando también el tono neutro pero con más proporción de valoración negativa (con porcentajes alrededor de hasta $53 \%$ en el caso de Peña Nieto en La Jornada, por ejemplo).

Respecto a la evolución de la cobertura informativa a lo largo de la campaña también se encontraron puntos que dan luz sobre el com- 
Esta revista forma parte del acervo de la Biblioteca Jurídica Virtual del Instituto de Investigaciones Jurídicas de la UNAM

portamiento de los medios, como se exhibe de forma precisa en las tablas 3 y 4 . Primero, se hizo evidente que Josefina Vázquez Mota quedó rezagada en cuanto al monto de cobertura recibida tras el primer mes de campaña, tanto en televisión como en prensa escrita. La cobertura hacia Gabriel Quadri de la Torre fue menor respecto de sus rivales y prácticamente sin valoración. Así, en los medios, el foco de atención se centró en sólo dos candidatos: Peña Nieto y López Obrador.

En relación con este último, cabe decir que recibió una cobertura menos favorable en televisión que los otros candidatos. Esta tendencia se incrementó conforme se acercaba el cierre de la campaña (con 30\% de notas negativas en el noticiero de Alatorre y $23.6 \%$ en el noticiero de López Dóriga). Asimismo, la postura de los medios electrónicos respecto al PRD se evidenció, pues fue el contendiente con menor cantidad de notas positivas ( $\tan$ solo una editorial). En cambio, en prensa escrita, aunque también aumentó su presencia en términos negativos conforme se llegó el final del proceso, López Obrador tuvo una cobertura más positiva comparada con la televisión, con clara preferencia en el caso del periódico La Jornada, donde el 31\% de las notas sobre él fueron en este sentido.

Por el contrario, el caso de Peña Nieto destaca porque en televisión fue el que tuvo mayor cobertura en términos positivos $(5.15 \%$ en TV Azteca; $4.48 \%$ en Televisa) aunque también tuvo efecto en términos negativos, que incrementó en mayo por la difusión del movimiento \#Yosoy132. Fue el que más apareció en notas de la prensa escrita, la cobertura a Peña Nieto en Reforma y La Jornada fue más y con mayor tendencia negativa rumbo al final de la campaña ( 45 y $51 \%$ en $L a$ Jornada y Reforma respectivamente en el último mes de campaña). En cambio, la información con valoración negativa sobre el priista disminuyó en El Universal durante junio (29\% de las notas).

Los hallazgos aquí presentados resultan importantes en un contexto de transición a la democracia que vive el país, por el papel que tienen los medios en este escenario, como se planteó en los objetivos de la investigación. Sin embargo, estos resultados se hicieron aún más relevantes por aspectos coyunturales particulares de la elección presidencial de 2012, pues el tema de la influencia de los medios de comunicación en el proceso se volvió central, primero por la nominación como candidato a un personaje que se consideraba fuertemente vinculado con Televisa, y luego con la aparición del movimiento \#Yosoy132. 
Esta revista forma parte del acervo de la Biblioteca Jurídica Virtual del Instituto de Investigaciones Jurídicas de la UNAM

\section{Medios de información y preferencias electorales}

Durante la campaña electoral 2012 en México, el Tecnológico de Monterrey realizó cinco encuestas ${ }^{14}$ de opinión pública vía teléfono celular, enfocadas principalmente a conocer la intención de voto, perfil sociodemográfico y hábitos informativos de la población mexicana en edad de votar. A partir de estas encuestas, es posible reconstruir los perfiles y hábitos informativos de diversos grupos de votantes. A continuación se destacan algunos de los hallazgos más relevantes.

¿Cómo se informó el electorado? De acuerdo con los resultados que arrojaron las encuestas, la televisión es el medio dominante de información política en términos absolutos, para todos los grupos educativos y de ingresos en México: tres de cada cuatro mexicanos la utiliza regularmente como fuente de información política. Sin embargo, el predominio de la TV es mucho más marcado entre los grupos con menor educación, quienes no sólo tienden a preferir la televisión en mayor medida, sino que también tienden a utilizarla como fuente exclusi$v a$ en una mayor proporción. En cambio, el uso de Internet es mucho más pronunciado entre quienes cuentan con educación universitaria y posgrado, alcanzando una magnitud comparable a la de la TV en estos grupos. En general, Internet es usado de forma complementaria por una buena parte de la población, principalmente aquella de mayores ingresos, alrededor de una tercera parte del electorado utiliza Internet para informarse de política. Por otra parte, los lectores de periódicos tienden a tener un mayor nivel educativo que la población en general, aunque los números totales de usuarios de estos medios son más reducidos, tan solo uno de cada siete mexicanos dice usar periódicos para informarse de política, y aunque uno de cada tres mexicanos dice utilizar Internet para informarse, generalmente lo hace de forma complementaria o secundaria con respecto a la televisión.

Tomados como conjunto, los noticieros de Televisa y TV Azteca son fuente de información política para el $70 \%$ y $80 \%$ de la población

14 Todas las encuestas son representativas a nivel nacional. Los detalles metodológicos y las tablas y gráficas que sirven de base para este artículo se pueden consultar en EGAP Gobierno y Política Pública, Proyecto "Observatorio de Medios y Opinión Pública”, http://www.observacionelectoral2012.com.mx/wp-content/uploads/2012/11/Info rmeFinal_EGAP.pdf. 
que declara informarse por dicha vía, que a su vez es una mayoría importante del electorado. Los noticieros de Televisa se sostienen como líderes de audiencia a lo largo de todos los segmentos de ingresos y de educación, lo que permite afirmar de forma inequívoca que es el grupo mediático más influyente en el país en términos de información política. En general, la televisión abierta y los noticieros nacionales dominan a los segmentos de menor educación, mientras que la televisión de paga o restringida representa una mayor competencia respecto de los grupos de la población con más nivel educativo. De hecho, ese aspecto de diferencia socioeconómica es revelador: la mayoría de la población se ubica en los segmentos de ingresos de bajo a mediobajo, y el nivel educativo del 70\% del electorado es igual o menor al de carrera técnica/preparatoria. Esto da una idea del mercado potencial sobre quienes los noticieros de televisión pueden influir: una mayoría de población de bajos recursos y bajo nivel educativo, con niveles de información presumiblemente menores. En síntesis, para la elección presidencial de México, la televisión fue el medio de información preferido en términos generales, con un dominio más marcado dentro de los estratos con menores ingresos y educación.

En lo que respecta a la audiencia de la prensa escrita, se pueden agrupar a los lectores de periódicos en dos grandes segmentos. Por una parte, están los lectores de periódicos locales y regionales, junto con periódicos de corte sensacionalista, que predominan en la categoría "otros". Dichos lectores tienden a pertenecer a los grupos de menores ingresos y educación. Por otra parte, se encuentran los lectores de periódicos de circulación nacional, que se consideran "de récord" y que tienden a apelar a los grupos de mayor nivel educativo y mejores ingresos. Entre los tres periódicos que se incluyeron en el análisis de medios de información presentado en la sección anterior, se aprecian perfiles de lectores algo similares. Los lectores de La Jornada tienden a estar concentrados geográficamente en el centro del país, mientras que los lectores de Grupo Reforma se concentran en las grandes zonas metropolitanas. Los periódicos de Grupo Reforma, en general, apelaron a un grupo de lectores con niveles de ingresos ligeramente superiores a los de La Jornada y El Universal, que cuentan con audiencias relativamente similares en este aspecto. Por otra parte, y conforme a lo que se esperaba, el impacto de estos diarios como fuentes de información política entre los segmentos de menores ingresos de la población fue mucho más limitado. 
Esta revista forma parte del acervo de la Biblioteca Jurídica Virtual del Instituto de Investigaciones Jurídicas de la UNAM

Finalmente, se puede destacar que los usuarios de Internet utilizan de forma predominante las redes sociales. Facebook, en particular, es mencionada como fuente de información política por una tercera parte de quienes declaran informarse a través de Internet. Tal y como se esperaba, el uso de Internet como fuente de información se encuentra fuertemente sesgado hacia los segmentos más jóvenes, urbanos, y de mayores niveles de ingreso y educación en la población del país. La gran mayoría de quienes se informan acerca de política por esta vía cuentan con estudios superiores a los de preparatoria; Internet de hecho aparece casi con los mismos niveles de mención que la televisión como fuente de información entre quienes cuentan con carrera universitaria o superior.

Si bien las cifras de usuarios que declaran utilizar Internet no son tan elevadas como las referentes a televisión, los usuarios de Internet declararon tener una mayor confianza en la información que reciben por esta vía. De acuerdo con los resultados del estudio, se puede apreciar que relativamente pocos usuarios de Internet recurren a fuentes de información "establecidas" para obtener información política (tales como las páginas de los periódicos o revistas especializadas). En cambio, la mayoría se informa a través de redes sociales (que pueden incluir canales oficiales de información por parte de candidatos y partidos) y de medios tales como YouTube. Sin duda, se requieren estudios con mayor énfasis en la naturaleza de los contenidos de información política que se consumen y comparten vía Internet para poder evaluar de una mejor manera el impacto que estos medios tuvieron para la campaña electoral.

\section{Preferencia electoral y medios de información}

Una vez establecidos algunos patrones generales de perfiles de audiencia para cada medio, se analizan a continuación la interacción entre las preferencias electorales y los medios de comunicación. En líneas generales, las preferencias electorales entre quienes declaraban utilizar la televisión como fuente de información no diferían marcadamente de los resultados globales para cada encuesta respectiva. Sin embargo, sí se aprecia que quienes mencionaron a la TV como su medio exclusivo de información política preferían a Enrique Peña 
Esta revista forma parte del acervo de la Biblioteca Jurídica Virtual del Instituto de Investigaciones Jurídicas de la UNAM

Nieto en mayor proporción. En otras palabras, aquellos usuarios que combinaban la televisión con otros medios de información tendían a repartir sus preferencias de forma más o menos representativa. En cambio, quienes declaraban no utilizar la televisión como fuente de información tendían a preferir de forma desproporcionada a Andrés Manuel López Obrador como presidente.

Este fenómeno parece estar relacionado con la conexión que existe entre los niveles de educación e ingreso con la preferencia de la TV como medio exclusivo de información. En líneas generales, Peña Nieto fue más exitoso en los segmentos de la población con menores ingresos y menor educación, que son justamente quienes tienden a depender de forma más exclusiva de la televisión (y en concreto la televisión abierta) como principal fuente de información. En contraparte, López Obrador fue más exitoso entre la población joven urbana con mayores niveles educativos; justamente el segmento del público que tiende a utilizar en menor medida la televisión como fuente de información política. Esta correspondencia entre la preferencia por un medio de información y un candidato es sumamente interesante, y otorga mayor solidez a la tesis que sostiene que los medios de información pueden influir de forma determinante en el "marco conceptual” o mental en el que la población forma sus decisiones políticas.

\begin{tabular}{|c|c|c|c|c|}
\hline Medio de información/candidato & $J V M$ & $E P N$ & $A M L O$ & GQT \\
\hline Resultado IFE & $26.1 \%$ & $39.2 \%$ & $32.4 \%$ & $2.3 \%$ \\
\hline Total encuesta EGAP ${ }^{1}$ & $25.2 \%$ & $38.9 \%$ & $32.3 \%$ & $3.6 \%$ \\
\hline Mencionan TV & $26.2 \%$ & $42.7 \%$ & $27.4 \%$ & $3.7 \%$ \\
\hline Únicamente $\mathrm{TV}^{2}$ & $24.9 \%$ & $48.3 \%$ & $23.6 \%$ & $3.2 \%$ \\
\hline No mencionan TV & $25 \%$ & $26.6 \%$ & $44.3 \%$ & $4 \%$ \\
\hline Únicamente Internet $^{3}$ & $22.9 \%$ & $16.7 \%$ & $57.8 \%$ & $2.6 \%$ \\
\hline Periódicos ${ }^{4}$ & $23.7 \%$ & $36.4 \%$ & $35.9 \%$ & $4.1 \%$ \\
\hline Radio $^{5}$ & $30.0 \%$ & $36.5 \%$ & $30.0 \%$ & $3.5 \%$ \\
\hline
\end{tabular}

1 Se aplicaron 2141 entrevistas a teléfonos celulares de ciudadanos en condiciones de votar entre el 25 y el 28 de junio de 2012. La muestra fue seleccionada de forma aleatoria y la responsable del levantamiento fue la doctora Olivia Carrillo, del departamento de Matemáticas del Tecnológico de Monterrey, Campus Monterrey; la aplicación se realizó a través del call center del Sorteo Tec, de la misma institución.

2 En este renglón se incluyen 928 entrevistados (43.3\% del total) que dicen informarse de las campañas electorales únicamente a través de la televisión.

3 En este renglón se incluyen 270 entrevistados (12.6\% del total) que dicen informarse de las campañas electorales únicamente a través del Internet. 
Esta revista forma parte del acervo de la Biblioteca Jurídica Virtual del Instituto de Investigaciones Jurídicas de la UNAM

\footnotetext{
${ }_{4}^{4}$ En este renglón se incluyen 368 entrevistados (17.2\% del total) que dicen informarse de las campañas electorales a través de los periódicos, aunque no necesariamente sin recurrir a algún otro medio, como pueden ser la televisión o Internet.

${ }_{5}$ En este rubro se incluyen 424 entrevistados (19.8\% del total) para los que dicen informarse de las campañas electorales a través de radio.
}

En la tabla anterior se respalda el argumento que se señalaba respecto a diferencias en las preferencias por candidato para quienes mencionan la televisión como fuente de información política, y se contrastan con las expresadas por quienes mencionan la TV exclusivamente (esto es, no mencionaron otros medios de información política), con los usuarios de Internet.

En la tabla se destaca, desde luego, la diferencia favorable que mantuvo Peña Nieto con López Obrador entre el público que usaba televisión como fuente de información política. Es notable el déficit que tiene López Obrador entre la audiencia de Televisa y TV Azteca, respecto a sus preferencias generales: de ocho puntos para TV Azteca, y de seis para Televisa. En cambio, Peña Nieto tiene un saldo favorable en Televisa de ocho puntos porcentuales con respecto a sus preferencias globales, y no tiene prácticamente diferencia en las otras cadenas de televisión. Asimismo, la preferencia por Josefina Vázquez Mota es 6 puntos mayor a su promedio global en las encuestas para TV Azteca, y dos puntos menor en Televisa y en el colectivo de las demás cadenas de televisión. El contraste es muy marcado con el público que declara que no utiliza la televisión como fuente de información política. En este grupo, la preferencia por López Obrador prácticamente duplica la expresada hacia Peña Nieto. La tendencia, muy distinta respecto a lo observado por la televisión, se encuentra sin duda relacionada con el uso de Internet y redes sociales como medio preferido por parte de los jóvenes urbanos y entre quienes contaban con mayores ingresos y niveles educativos. En estos segmentos, la preferencia por Andrés Manuel López Obrador fue más pronunciada.

En términos generales, se aprecia una relación entre las preferencias de votación y el uso de la televisión como fuente de información. La tendencia de la audiencia televisiva de preferir a Peña Nieto sin duda merece ser analizada con mayor profundidad. En la sección respectiva de este artículo se ha establecido que los noticieros de televisión habían presentado, en términos generales, una cierta preferencia por Peña Nieto en volumen de cobertura y tendencias de nota, sin embargo, ello no garantiza la influencia que se observa en las prefe- 
Esta revista forma parte del acervo de la Biblioteca Jurídica Virtual del Instituto de Investigaciones Jurídicas de la UNAM

rencias electorales. Es posible que, como se ha sugerido en investigaciones semejantes en otros países, la audiencia simplemente consume los medios que mejor reflejan sus preferencias. En otras palabras, los votantes de Peña Nieto tienden a ver televisión abierta por sus condiciones socioeconómicas y sus preferencias políticas, por otra parte, no puede descartarse una relación de reforzamiento mutuo, que el público con menor nivel de información y educación es más susceptible a manipulación, y más vulnerable a "absorber" los sesgos informativos detectados y transformarlos en actitudes políticas.

\section{Grupos de enfoque ${ }^{15}$}

Como complemento a la investigación, también durante el periodo electoral se configuró un proyecto de corte cualitativo consistente en la realización de 21 grupos de enfoque llevados a cabo en las tres principales ciudades del país: Monterrey, Distrito Federal y Guadalajara. El desarrollo de los mismos fue en dos etapas: en la primera, participaron 12 grupos en los meses de mayo y junio de 2012 (antes de las elecciones presidenciales), $y$, en la segunda, 9 grupos durante la semana posterior a las elecciones del 1o. de julio. El objetivo principal era analizar el impacto de los medios de comunicación, a través de espacios noticiosos y los spots publicitarios de las campañas electorales presidenciales de 2012, en las opiniones de los votantes sobre los candidatos y la influencia que éstos tuvieron en la decisión final de su voto. A continuación se presentan algunas de las principales observaciones y conclusiones generales a las que se llegaron.

\section{Televisión}

El medio de comunicación más importante para informarse sobre las campañas presidenciales de 2012 en México fue la televisión a través

15 El Proyecto de grupos de enfoque lo coordinó la candidata a doctora en Ciencias Sociales, Gabriela Salazar, quien además moderó las sesiones de Monterrey. Los detalles de dicho estudio se pueden encontrar en: $h t t p: / / w w w . o b s e r v a c i o n e l e c t o r a l 2012 . c o m . m x /$ wp-content/uploads/2012/11/InformeFinal_EGAP.pdf, consultada el 2 de febrero de 2013. 
Esta revista forma parte del acervo de la Biblioteca Jurídica Virtual del Instituto de Investigaciones Jurídicas de la UNAM

de los spots publicitarios de cada candidato y de los noticieros. Existe gran preferencia por Televisa y Joaquín López Dóriga en comparación con TV Azteca y Javier Alatorre.

La imparcialidad de los noticieros de televisión fue cuestionada por todos los grupos (en mayor o menor medida). Principalmente se cuestionó la imparcialidad de Televisa y el noticiero de Joaquín López Dóriga; sin embargo, también TV Azteca y Javier Alatorre fueron cuestionados, aunque en menor proporción (se sintonizaban con menor frecuencia). En general se considera que Televisa y Joaquín López Dóriga, favorecen a EPN y al PRI.

El estudio indica que la televisión y los spots, sí llegaron a influir significativamente en la decisión de los votantes no partidistas, al construir a partir de dichos comerciales, una percepción muy definida y la imagen de cada contendiente. En cambio, para los votantes partidistas del PRI y del PRD, la televisión solamente sirvió para confirmar su decisión de voto hacia su partido y candidato. En este sentido, se podría concluir que la televisión no fue un factor decisivo en el voto de estas personas; ya que ellos no cambiarían el sentido de su voto - ni lo cambiaron - por la campaña negativa contra su respectivo candidato. Por otra parte, el caso de los votantes partidistas del PAN es especial debido a que a la mayoría de los panistas no les convencía demasiado su candidata, su imagen y mensaje, tampoco sus spots televisivos. En general, su identificación y lealtad estaba dirigida más hacia el PAN que a JMV.

\section{Prensa escrita}

La prensa, aunque es un medio muy poco utilizado, es considerada en general, como un medio confiable. Son muy pocas las ocasiones en que los participantes suponen que los periódicos son parciales.

Los grupos que más leen periódicos son los de nivel socioeconómico medio alto y las personas de mayor edad (más de 40 años). En cambio, los grupos que menos leen periódicos son los jóvenes, con excepción de periódicos independientes que encuentran en Internet y en redes sociales. Las personas de estratos socioeconómicos bajos llegan a leer periódicos de nota roja (amarillistas) como Metro y Express en Monterrey o Metro y Gráfico en el Distrito Federal. Los periódicos mencionados son más baratos y son considerados más sencillos para leer. 


\section{Internet}

El Internet -en especial las redes sociales-irrumpió en la campaña presidencial de 2012 como un fuerte medio de comunicación que compitió con la televisión. Los grupos que más utilizaron Internet fueron los jóvenes y las personas de nivel socioeconómico medio y medio alto, y, a nivel partidista, los panistas. Es importante destacar que las redes sociales Facebook y Twitter fueron los espacios virtuales más usados para dar seguimiento a las campañas presidenciales. Existe una tendencia a considerar que Internet - sobre todo las redes sociales y YouTube- es un medio más confiable, debido a que suponen, no se puede manipular la información que se publica (o es muy difícil). En Internet hay gran pluralidad de ideas, de opiniones, de participantes. Son utilizados por mucha gente (en cantidad y en diversidad). Todo aquel que desee compartir algún tipo de información, puede hacerlo. No hay censura, hay mayor cobertura y más acceso. Es más ágil (gracias a los smartphones) y es instantáneo (tiempo real), se actualiza constantemente a lo largo del día.

Finalmente, como se señalaba con antelación, el uso de Internet está más relacionado con la edad y nivel socioeconómico que con la afiliación o preferencia partidista.

\section{Los familiares, amigos y conocidos: las relaciones sociales}

Los familiares, amigos y demás gente de confianza, siguen representando una fuente importante de información con respecto a las campañas electorales y, además, de gran influencia a la hora de decidir por quién votar. De manera general, esta opinión es para la mayoría, incluso más importante que los propios medios de comunicación.

Los priistas fueron el único grupo de identidad partidista que mencionó la asistencia a eventos organizados por el partido y sus candidatos; así como a reuniones vecinales y seccionales.

\section{Guerra sucia}

Todos los grupos, partidistas y no partidistas coinciden en que la campaña negativa estuvo enfocada principalmente en contra de EPN. Exis- 
Esta revista forma parte del acervo de la Biblioteca Jurídica Virtual del Instituto de Investigaciones Jurídicas de la UNAM

te un desagrado generalizado hacia la guerra sucia. Josefina Vázquez Mota fue considerada como la candidata que más utilizó este tipo de campaña, seguida por Andrés Manuel López Obrador. Enrique Peña Nieto, por su parte, fue percibido como un candidato enfocado en sus propuestas y que no recurrió a la guerra sucia.

\section{Percepción de los candidatos}

Para finalizar, se incluye una síntesis de los principales atributos o defectos que los participantes de los grupos de enfoque tradujeron de cada candidato a partir de la información obtenida a través de los distintos medios de comunicación analizados.

JVM, positivos: $a$ ) continuidad: PAN mejor que PRI. Negativos: a) enfocada a criticar a EPN; b) falsa, hipócrita, no auténtica; $c$ ) se escudaba en "ser mujer"; $d$ ) no causan impacto (confusos, muchos temas); e) insegura/nerviosa/falta de carácter; $f$ ) mensaje confuso: diferente, $y g$ ) mal manejo de campaña.

EPN, positivos: a) mejor producción de spots publicitarios. Mejor equipo de campaña; $b$ ) mejor mercadotecnia; $c$ ) nunca atacó/ propositivo; $d$ ) se enfocaba en hacer propuesta; e) firmeza y seguridad, y f) compromisos concretos. Negativos: $a$ ) muy enfocado en su imagen personal; b) actor de telenovela "cara bonita"; c) falta de cultura; d) no espontáneo "de libreto"; e) producto creado: mercadotecnia, y f) ligado a Televisa y a TV Azteca.

AMLO, positivos: $a$ ) humano; $b$ ) cercano a la gente; $c$ ) diferente al 2006/conciliador (más positivos), y $d$ ) representaba el cambio. Negativos: $a$ ) Violento; $b$ ) alborotador de masas; $c$ ) carácter inestable; d) mesiánico; e) socialista/Chávez/Cuba; f) populista, y g) recuerdo de la reacción de 2006 en sentido negativo.

GQT, positivos: $a$ ) buena participación en el primer debate. Negativos: $a$ ) chistosos/ raros/ cómicos; $b$ ) no presenta propuestas concretas; c) insignificante "de relleno", y d) poco conocido.

\section{Conclusiones}

Al observar los resultados del análisis de la cobertura de los medios (del framing y del tono, por una parte) y de lo referente a la opinión 
Esta revista forma parte del acervo de la Biblioteca Jurídica Virtual del Instituto de Investigaciones Jurídicas de la UNAM

pública (con las encuestas y grupos de enfoque, por otra), es posible identificar cómo los conceptos que proyectan los medios de comunicación - con respecto a los candidatos, partidos políticos y campañas electorales - son los mismos que los ciudadanos, que consumen estos medios, perciben y asimilan. Existe una clara afinidad entre las apreciaciones de los electores y las tendencias o sesgos de los medios de comunicación.

La revisión minuciosa que generó el Observatorio permite identificar que todos los medios muestran un sesgo a favor de alguno o en contra de uno o más candidatos; estas tendencias se perfilaron desde un año antes del inicio de las campañas electorales y, en todos los casos, son perceptibles los cambios de intensidad en las mismas a lo largo de dicho periodo.

En el caso de las televisoras, el apoyo al candidato que finalmente resultó ganador de la elección se hace más notorio en los meses previos al inicio del proceso de selección interna de los partidos y, posteriormente, en el último mes de campaña. En los medios impresos, las tendencias se hacen más evidentes, con marcadas diferencias entre sí, lo que incluso permite identificar claramente un periódico que se posiciona a favor de AMLO, y dos periódicos que incrementan sus críticas en contra de alguno de los candidatos, uno contra quien ganó la elección y el otro contra el segundo lugar.

En la televisión, pudimos observar una "visión unificada" en donde, independientemente de la televisora - sea Televisa o TV Azteca- el tono y el framing de la cobertura fueron muy similares y presentaron el mismo enfoque.

En la prensa escrita, por su parte, cada uno de los periódicos analizados proyecta tonos y framings diferentes; a su vez, éstos son elegidos por los lectores que, por lo general, simpatizan con las ideologías de cada uno. Los ciudadanos poseen opciones diversas de periódicos y puede elegir cuál es el que desean leer — un universo más amplio-.

Retomando lo que se considera el hallazgo central de esta investigación, es posible decir que en medios impresos sí se percibe claramente el pluralismo externo en la información que se divulga, contrario a lo que sucede en el caso de las televisoras en las que prevalece la uniformidad en la cobertura informativa y, por ende, no existe ningún tipo de pluralismo ni interno ni externo.

Los resultados de las encuestas de preferencia electoral muestran la correlación entre el medio de información (tv, prensa, radio, Inter- 
Esta revista forma parte del acervo de la Biblioteca Jurídica Virtual del Instituto de Investigaciones Jurídicas de la UNAM

net) y las preferencias electorales, agudizado entre los que se informan únicamente a través de la televisión y los que lo hacen por Internet. Con la información disponible no es viable atribuir la causalidad a la información que los medios proporcionan, pues como se señaló en el marco teórico, la influencia de éstos está mediada por una multitud de factores, además que la causalidad también puede ser inversa, es decir, las audiencias seleccionan sus medios en función de su identificación con ellos.

Pero sí puede afirmarse que en los medios con pluralidad externa las preferencias electorales se dispersan más equilibradamente entre los distintos candidatos, que en aquéllos que carecen de esta, donde los votos se concentran en el candidato favorecido por los mismos.

Y como es precisamente la televisión (medio sin ningún tipo de pluralidad) la que contribuye en mayor medida a informar y, por ende, construir la percepción del ciudadano sobre los candidatos, la ausencia de dicha pluralidad genera desigualdad en la contienda que resulta muy lesiva para la competencia electoral y, en general, para la democracia.

\section{Fuentes consultadas}

Cohen, B., The Press and Foreign Policy, Princeton, NJ, Princeton University Press, 1963.

DAHL, Robert A., La igualdad política, Buenos Aires, Fondo de Cultura Económica de Argentina, 2008.

Diamond, Larry y Morlino, Leonardo, “An Overview”, The Quality of Democracy. Journal of Democracy $(15,4), 2004$, pp. 20-31.

Domke, D et al., "Media Priming Effects: Accessibility, Association, and Activation", Communications abstracts, 21( 6), 1998.

Esser y D’Angelo, "Framing the Press and Publicity Process in U.S., British and German General Election Campaings: A comparative Study of Metacoverage", The Harvard International Journal of Press/ Politics 11(44), 2006.

Fairhurst, G. y Star, R., The Art of Framing, San Francisco, JosseyBass, 1996.

Gerbner, G., y Gross, L., “The Scary World of TV's Heavy Viewer”, Psychology Today, 10(4), 1976. 
Esta revista forma parte del acervo de la Biblioteca Jurídica Virtual del Instituto de Investigaciones Jurídicas de la UNAM

Hallin, Daniel C. y Mancini, Paolo, Comparing Media Systems. Three Models of Media and Politics, Cambridge, USA, Cambridge University Press, 2004.

Katz, E., y Lazarsfeld, P., Personal Influence, Nueva York, The Free Press, 1955.

McСомвs, M. E. y Shaw, D., "The Agenda-Setting Function of Mass Media", $P O Q$, 36; 1972.

Scheufele, D. A., Agenda-Setting, Priming, and Framing Revisited: Another Look at Aognitive Effects of Political Communication, Communication abstracts, 24(1), 2001.

Semetko y Valkunberg, "Framing European Politics: A Content Analysis of Press and Television News", Journal of Communication, 50 (2), 2006.

Tichenor, P.J. et al., "Mass Media Flow and Differential Growth in Knowledge”, Public Opinion Quarterly 34, Colombia, University Press, 1970. 